Georgian Mathematical Journal

Volume 8 (2001), Number 1, 97-109

\title{
ON LOCAL INVARIANTS OF TOTALLY REAL SURFACES
}

\author{
G. KHIMSHIASHVILI
}

\begin{abstract}
A class of totally real surface singularities is introduced for which one can define local topological invariants. The main result yields an algebraic estimate for the self-intersection index of a totally real germ. It is derived from an algebraic upper estimate for the index of a quasi-homogeneous vector field which generalizes the previously known estimates in terms of the so-called Petrovsky numbers.
\end{abstract}

2000 Mathematics Subject Classification: 32S05, 55M25.

Key words and phrases: Totally real surface, linking number, self-intersection index, local algebra, signature technique, Petrovsky numbers.

\section{INTRODUCTION}

We deal with germs of totally real two-dimensional surfaces with isolated singularities in $\mathbf{C}^{2}$ given by polynomial parametrizations. Such germs possess two basic topological invariants - the self-intersection index and the Maslov index [15], [6], - which contain important geometric information [9], [11]. In this context, one of the crucial problems is to develop effective methods for computing these invariants in terms of algebraic data of parametrizing polynomials. Another natural problem is to obtain effective estimates for possible values of these topological invariants on various classes of such germs.

In the present paper we obtain some results in this direction which are basically applicable to the self-intersection indices investigated in [6], [9], [11]. For simplicity, we only deal with germs of totally real surfaces in $\mathbf{C}^{2}$, but our approach is apparently meaningful in more general settings. Main attention is be given to germs which can be parametrized by quadruples of homogeneous polynomials and satisfy some mild non-degeneracy condition (we call them "robust totally real germs"). Such germs appear in many aspects of symplectic geometry [6] and complex analysis [15], [9] (in fact, all examples considered in [6], [15], [9] belong to this class) so they deserve some attention in themselves.

The main result (Theorem 2) yields an upper estimate for the absolute value of the self-intersection index in terms of degrees of parametrizing polynomials. Its proof relies on an algebraic method of computing self-intersection indices by means of the so-called signature formulae for the topological degree [4], [10] which involve rather specific algebraic notions and constructions. In order to make the exposition more clear and self-contained, we describe this algebraic technique in some detail and then explain how to use it for our purposes.

ISSN 1072-947X / \$8.00 / (c) Heldermann Verlag www.heldermann.de 
We start by discussing indices (local topological degrees) of isolated singular points of polynomial vector fields [2]. In particular, we obtain an algebraic estimate for the index of a quasi-homogeneous vector field (Theorem 1) in terms of analogues of the so-called Petrovsky numbers [1]. This estimate generalizes similar results from [10] and [1] and plays the crucial role in our approach. It should be noted that quasi-homogeneous vector fields inevitably arise in our considerations and we need this estimate in exactly such generality. Theorem 1 is related to some interesting problems. For example, in many cases this estimate turns out to be exact but in general the question of its exactness remains open.

The definition and relevant properties of "robust" totally real surface germs with isolated singularities are presented in the third section. In particular, we introduce the (local) self-intersection index of a totally real germ in the spirit of [9] and explain how can one compute it using the index formulas from [5], [9]. Since these index formulas involve the notion of the Maslov index, for the sake of clarity we give definition of the latter and mention its main properties.

In the last section we formulate the main result and describe its proof. In conclusion, we briefly mention some possible generalizations and related results.

\section{INDICES OF POLYNOMIAL VECTOR FIELDS}

Let $\mathbf{R}_{n}$ denote the ring of polynomials in $n$ indeterminates with real coefficients. A collection of polynomials $f_{1}, \ldots, f_{m} \in \mathbf{R}_{n}$ apparently defines a polynomial mapping $f: \mathbf{R}^{n} \rightarrow \mathbf{R}^{m}$. For $m=n$ one obtains a polynomial endomorphism of $\mathbf{R}^{n}$ which we will regard and address as a polynomial vector field on $\mathbf{R}^{n}$.

Assume also that we are given a collection of positive rational numbers $w=\left(w_{1}, \ldots, w_{n}\right)$ which are considered as weights of indeterminates $x_{1}, \ldots, x_{n}$. Then the $w$-degree of any monomial $\prod x_{i}^{k_{i}}$ is defined as $\sum k_{i} w_{i}$ and a polynomial $P \in \mathbf{R}_{n}$ is said to be quasi-homogeneous (qh) of $w$-degree $k$ if $w$-degrees of all monomials contained in $P$ with non-zero coefficients are equal to $k$ [2].

A polynomial mapping $f$ is called a quasi-homogeneous mapping of $w$-degree $d=\left(d_{1}, \ldots, d_{m}\right)$ if each of its components $f_{i}$ is a quasi-homogeneous polynomial of $w$-degree $d_{i}$ (cf. [2]). As a shorthand we will sometimes say that $f$ is of qhtype $(w, d)$.

For $m=n$ we obtain a notion of quasi-homogeneous vector field. Such a qh vector field is called non-degenerate if the origin is its isolated zero, which is equivalent to requiring that polynomials $f_{i}$ have no non-trivial common zeros in $\mathbf{R}^{n}$.

As is well known, for every vector field $f$ having the origin as its isolated zero, its (topological) index $\operatorname{ind}_{0} f$ at the origin is defined as

$$
\operatorname{ind}_{0} f=\operatorname{deg}\left\{f /\|f\|: S_{r}^{n-1} \rightarrow S_{1}^{n-1}\right\},
$$

where $S_{r}^{n-1}$ denotes the sphere of radius $r$ centered at the origin, and deg stands for the usual topological degree of a continuous mappings between two compact 
oriented ( $n-1)$-manifolds [16]. Here, of course, the positive number $r$ is assumed to be sufficiently small, which guarantees that the value of topological degree in the right-hand side does not depend on $r$.

In this section we will be concerned with estimating local indices of nondegenerate qh vector fields of fixed qh-type $(w, d)$ in terms of weights $w$ and degrees $d$.

It turns out that effective and useful estimates are provided by qh-analogues of the so-called Petrovsky numbers introduced in [1]. In order to define them, we introduce an integer multi-index $k=\left(k_{1}, \ldots, k_{n}\right) \in \mathbf{Z}^{n}$ and put:

$$
P(n ; w, d)=\operatorname{card}\left\{k \in \mathbf{Z}^{n}, 0 \leq k_{i} \leq d_{i}: \sum w_{i} k_{i}=\sum\left(d_{i}-w_{i}\right) / 2\right\} .
$$

Here card stays for the number of elements of a finite set. Thus this formula expresses the number of integer points lying on the indicated hyperplane inside a certain parallelepiped defined by weights and degrees. Apparently, these numbers may be effectively computed for any concrete values of "parameters" $n, w, d$. Their asymptotics and behaviour for "sufficiently large" values of parameters suggest some interesting questions but this is beyond the scope of present paper.

Now we are ready to formulate the main result of this section.

Theorem 1. Let $f$ be a non-degenerate quasi-homogeneous vector field on $\mathbf{R}^{n}$ of type $(w, d)$. Then the absolute value ot its local index does not exceed the corresponding Petrovsky number

$$
\left|\operatorname{ind}_{0} f\right| \leq P(n ; w, d)
$$

In order to prove this estimate we need some constructions and notation related to local algebras of polynomial endomorphisms (vector fields) which we borrow from [4] and [10]. Before giving the proof of Theorem 1 we present some preparatory comments.

Given a non-degenerate vector field $f$ as above, its local algebra at the origin is defined as $A=A_{0}(f)=\mathbf{R}_{n}[[x]] /(f)$, where $\mathbf{R}_{n}[[x]]$ denotes the ring of formal power series in $n$ indeterminates with real coefficients, and $(f)$ denotes the ideal generated by components of $f$. Let us also denote by $J_{f}$ the Jacobian (determinant of the Jacobi matrix) of $f$ and by $j$ its class in $A$. As is well known, $j$ is a non-zero element of $A[8]$.

According to [4] and [10], the index of $f$ at the origin may be computed using the so-called Gorenstein quadratic form $Q_{f}$ on the local algebra $A$. In order to define it, one first chooses a $\mathbf{R}$-linear functional on $A$ satisfying the condition $l(j)>0$. Then, for $a \in A$, set

$$
Q_{f}(a)=l\left(a^{2}\right)
$$

It turns out that $Q_{f}$ is a non-degenerate quadratic form on $A$ (this is a nontrivial fact which may be derived from the Grothendieck local duality theorem 
[10]) and, moreover, one has

$$
\operatorname{ind}_{0} f=\operatorname{sig} Q_{f},
$$

where sig denotes the usual signature of a non-degenerate quadratic form, i.e., the difference between numbers of positive and negative squares in its diagonal form.

This equality gives a lot of information about ind $\mathrm{d}_{0} f$ and, in particular, implies some general algebraic estimates for $\left|\operatorname{ind}_{0} f\right|$ [4], [10]. Indeed, if $\mu=\operatorname{dim}_{\mathbf{R}} A$ is the (algebraic) multiplicity of $f$ at the origin [2], then formula (4) apparently implies that $\left|\operatorname{ind}_{0} f\right| \leq \mu$, and this estimate, despite its simplicity, is useful because the multiplicity is algorithmically computable from the coefficients of $f[2]$.

In the qh case one can obtain another simple estimate which is also much weaker than the one given in Theorem 1 . We give it because it is simple and may be easily derived from the known results.

\section{Corollary 1.}

$$
\left|\operatorname{ind}_{0} f\right| \leq\left(\prod \frac{d_{i}}{w_{i}}\right)^{1-\frac{1}{n}}
$$

This follows from an "inequality of Minkowski type" having the form $\left|\operatorname{ind}_{0} f\right| \leq \mu^{1-\frac{1}{n}}$ which was established in the Appendix to [4] and from the well-known fact that the multiplicity of a qh vector field is equal to the product of degrees divided by the product of weights [2].

From now on we assume that $f$ is non-degenerate of qh-type $(w, d)$. We make use of the fact that the structure of local algebras of such mappings was thoroughly studied in [2], [7]. So we freely borrow the necessary results from these two sources. Granted that, the proof of Theorem 1 is obtained by making several observations which we formulate as lemmas.

Proof of Theorem 1. First of all, in the qh case the local algebra $A$ can be equipped with a natural grading depending only on $w$ and $d[2]$. It is induced from a natural grading on $\mathbf{R}_{n}[[x]]$ defined by $w$-degrees of monomials. This suggests that some properties of $f$ can be properly described by choosing a $w$ quasi-homogeneous basis in $A$ and counting basis elements with fixed $w$-degrees.

Indeed, according to [7], the Poincaré polynomial of $A$ is completely determined by weights and degrees. This means that, for any such $f$ of qh-type $(w, d)$ and any quasi-homogeneous basis in $A_{0}(f)$, the number of basis elements of fixed $w$-degree is always the same. In particular, one can formulate two important statements about the structure of graded algebra $A$ which can be verified using the explicit formula for Poincaré polynomial of $A$ given in [7]. Actually, both these statements are well known and we present them exclusively for the sake of completeness.

Lemma 1. The $w$-degree of any quasi-homogeneous element in $A$ does not exceed $d_{\max }=\sum\left(d_{i}-w_{i}\right)$ which is exactly the $w$-degree of Jacobian $J_{f}$. 
In other words, its class $j$ is the "highest" element in $A$ (in an evident geometric sense which refers to plotting monomials on the integer lattice $\mathbf{Z}^{n}$ ). Apparently, one can always choose a basis of $A$ consisting of (classes of) quasihomogeneous polynomials. In particular, one can assume all elements of the basis to be monomials (the so-called monomial basis [2]).

Lemma 2. Every quasi-homogeneous basis of $A$ (e.g., a monomial basis) contains exactly one element of $w$-degree $d_{\max }$, in particular, one can always assume that $j$ is the unique basis element of weight $d_{\max }$.

Now we can prove two lemmas of more technical nature.

Lemma 3. For any quasi-homogeneous basis element a of $w$-degree greater than $d_{\max } / 2$, one has $Q_{f}(a)=0$.

Indeed, the algebra $A$ is graded so the element $a^{2}$ has the $w$-degree greater than $d_{\max }$, which, in virtue of Lemma 1 , implies that it is a zero class in $A$.

Denote now by $I$ the ideal generated by all basis elements of $w$-degree greater than $d_{\max } / 2$. From Lemma 1 it follows that $I^{2}=0$ in $A$. Consider now its annihilator ann $I$. Then it is clear that $I \subset$ ann $I$.

Lemma 4. The ideal ann $I$ does not contain any basis element of $w$-degree smaller than $d_{\max } / 2$.

In order to prove this lemma, we notice that from the Grothendieck local duality theorem [8] it follows (and this fact is actually equivalent to this theorem) that, for every non-zero element $a \in A$ there exists an element $b \in A$ such that $a b=j$ (so one can write $b=j / a$ ). Now for any quasi-homogeneous element (e.g., basis monomial) of degree smaller than $d_{\max } / 2$, the element $j / a$ has the degree not less than $d_{\max } / 2$, hence it belongs to $I$, which would contradict the assumption that $a$ belongs to ann $I$.

This enables us to prove our last lemma which is crucial for the proof of Theorem 1.

Lemma 5. The factor-space ann $I / I$ is spanned by basis elements of $w$ degree $d_{\max } / 2$.

Let us denote by $p$ the number of basis elements of $w$-degree $d_{\max } / 2$. Of course it may happen that not all of them are needed to span this factor but in any case we have the inequality

$$
\operatorname{dim}_{\mathbf{R}} \text { ann } I-\operatorname{dim}_{\mathbf{R}} I \leq p .
$$

Now let us notice that using some standard facts about non-degenerate quadratic forms, from formula (2) it follows (and this fact is explicitly stated in [4], [10]) that

$$
\left|\operatorname{ind}_{0} f\right|=\left|\operatorname{sig} Q_{f}\right|=\operatorname{dim}_{\mathbf{R}} \text { ann } M-\operatorname{dim}_{\mathbf{R}} M,
$$


where $M$ is any ideal of $A$ which is maximal among the ideals of $A$ having the zero square. Of course we can choose $M$ such that $I \subset M$. Then it is evident that

$$
\operatorname{dim}_{\mathbf{R}} \text { ann } M-\operatorname{dim}_{\mathbf{R}} M \leq \operatorname{dim}_{\mathbf{R}} \text { ann } I-\operatorname{dim}_{\mathbf{R}} I
$$

and we finally get

$$
\left|\operatorname{ind}_{0} f\right| \leq \operatorname{dim}_{\mathbf{R}} \text { ann } I-\operatorname{dim}_{\mathbf{R}} I .
$$

We conclude that $\left|\operatorname{ind}_{0} f\right| \leq p$, where the number $p$ is the same as in formula (7). In order to finish the proof, it remains to notice that from the results of [7] it follows that this number $p$ cannot exceed the Petrovsky number $P(n ; w, d)$ introduced above.

Analyzing this proof it is not difficult to derive from Lemma 5 a simpler (but of course much weaker) estimate in the case where the origin is a degenerate (multiple) zero of the vector field $f$ (as is well known, this takes place if and only if $\left.\mu=\operatorname{dim}_{\mathbf{R}} A \geq 2[2]\right)$.

Corollary 2. If the origin is a degenerate zero of a $(d, w)$-qh field $f$, then

$$
\left|\operatorname{ind}_{0} f\right| \leq \frac{1}{2} \prod \frac{d_{i}}{w_{i}}
$$

It is also useful to have a geometric reformulation of our estimate in the case of a homogeneous vector field (i.e., when all $w_{i}=1$ ).

Corollary 3. If $f$ is a homogeneous vector field of multi-degree $d$ in $\mathbf{R}^{n}$, then $\left|\operatorname{ind}_{0} f\right|$ does not exceed the number of integer points lying inside the parallelepiped $\Pi\left[0, d_{i}\right]$ on the hyperplane orthogonal to its main diagonal and passing through its center.

An especially important and instructive case is when $f$ is the gradient of a homogeneous $d$-form. Then one has to consider the central section of a cube $[0, d-1]^{n}$ and it is quite a visual exercise to compute these numbers for $n=3$ and small values of $d$. Some interesting examples of such calculations in the homogeneous case are presented in [12]. The qh-case seems to be more tricky so one may hope to encounter some interesting phenomena by computing these numbers for various qh-types.

Remark 1. In concrete cases one can also obtain a useful estimate for $\left|\operatorname{ind}_{0} f\right|$ by computing the number of basis elements of $w$-degree $d_{\text {max }} / 2$ from the explicit formula for the Poincaré polynomial presented in [7].

Remark 2. It would be interesting to establish if this estimate is exact on the set of all qh vector fields of fixed $(w, d)$-type. In many cases this can be established by using examples of the vector fields constructed by Khovanskii [12] who showed that a similar question (in a global setting) for homogeneous vector fields on $\mathbf{R}^{n}$ had a positive answer. However, the author was unable to solve this question in general. 
Remark 3. It is easy to see that in all non-trivial cases this estimate is better than the one given in Corollary 1. It would be interesting to make a thorough comparison of the arising estimates with the estimates for global indices (winding numbers) of polynomial vector fields obtained in [12].

In the sequel we need a slight generalization of Theorem 1. Recall that a vector field $g$ is called a semi-quasi-homogeneous (sqh) field of type $(w, d)$ if $g_{j}=f_{j}+h_{j}, j=1, \ldots, n$, where $f_{j}$ define a non-generate qh field of type $(w, d)$ and, for every $j$, the polynomial $h_{j}$ contains only monomials of $w$-degree greater than $d_{j}[2]$.

Corollary 4. For any sqh vector field $g$ of type $(w, d)$ on $\mathbf{R}^{n}$,

$$
\left|\operatorname{ind}_{0} f\right| \leq P(n ; w, d) .
$$

The corollary follows from the well known fact that both the local index and the Poincaré polynomial of such a sqh vector field $g$ coincide with those of its "initial" qh-part $f[7]$.

\section{Invariants of Totally Real Germs}

We will work with certain germs of real two-dimensional algebraic subsets in $\mathbf{C}^{2}$. More precisely, we will consider germs $X \subset \mathbf{C}^{2}$ which can be defined as common zeroes of several polynomials on $\mathbf{R}^{4}$ (regarded as "realification" of $\mathbf{C}^{2}$ ) and satisfy some mild tansversality condition.

In order to investigate certain properties of such germs which are of interest in complex analysis (in particular, the existence of complex tangents and attached analytic discs [5], [11]), we need to consider the grassmanian $G=G r_{+}(2,4)$ of oriented two-planes in $\mathbf{R}^{4}$. As is well known, $G$ is a compact four-dimensional smooth manifold diffeomorphic to $S^{2} \times S^{2}$.

In the sequel it will be important to distinguish between the complex lines in $\mathbf{C}^{2}$ and the so-called totally real planes in $\mathbf{C}^{2}$, so we recall that a twodimensional plane in $V \in G$ is called totally real if $V+\mathbf{i} V=\mathbf{C}^{2}$. We denote by $G_{R}$ the subset of $G$ consisting of totally real planes.

The subset of all complex lines taken with their natural (complex) orientations is homeomorphic to $\mathbf{C P}^{1}$ and is denoted by $P_{+}$. Another diffeomorphic copy $P_{-}$ of $\mathbf{C P}^{1}$ lying in $G$ is obtained by taking all complex lines with the orientation opposite to the complex one. Thus the collection $G_{C}$ of all oriented complex lines is the union of two disjoint spheres $S^{2}$ smoothly embedded in $G$. These and other submanifolds of $G$ are always endowed with their natural complex orientation.

Definition 1. ([11]) A germ $X$ of a real two-dimensional variety in $\mathbf{C}^{2}$ is called a robust totally real germ (ROTORG) if it is regular outside its basepoint, has transversal intersections with all sufficiently small spheres around the basepoint, and, for every regular point $p \in X$, the tangent plane $T_{p} X$ is a totally real plane. 
Such objects are easily seen to exist and below we indicate several sources of examples. Similar definitions appeared earlier in [9] and [11]. The present version seems more convenient for our concrete purposes.

Remark 4. Such a germ may have (at most) an isolated singularity (in the usual sense of algebraic geometry [8]) at the basepoint, in which case one can speak of a "totally real singularity" (TORS) and think of it as a small piece of a totally real surface with an isolated singularity.

Remark 5. By comparing Definition 1 with the definition of germs of generic immersions of surfaces in $\mathbf{C}^{2}$ given in [9], it becomes evident that our notion is less restrictive. So examples of generic immersions of [9] and [5] provide us with a plenty of ROTORGs. Actually, these two definitions are very close but it seemed more natural for us to consider totally real subvarieties without referring to explicit parametrizations.

Examples of ROTORGs naturally arise in complex analysis and symplectic geometry, e.g., as singularities of generic Lagrangian immersions [6]. Usually, they appear as parametrized (uniformized) germs, with quasi-homogeneous parametrizing polynomials. This is precisely the setting suitable for our purposes, so in the rest of the paper we assume that the germ $X$ is defined by a (germ of) quasi-homogeneous polynomial mapping $\mathbf{R}^{2} \rightarrow \mathbf{R}^{4}$.

By applying some standard technique of singularity theory, it is possible to show that "most of" (or generically) such germs are robust in the sense of Definition 1. For the sake of brevity, we will simply assume that $X$ is a ROTORG given as the image of a quasi-homogeneous mapping as above.

A typical example is provided by the so-called "unfolded Whitney umbrella" [6] which is defined as the image of mapping

$$
W: \mathbf{R}^{2} \rightarrow \mathbf{R}^{4}, \quad(x, y) \mapsto\left(x^{2}, y, x y, \frac{2 x^{3}}{3}\right),
$$

and plays an important role in the theory of Lagrangian immersions [6], [9].

Another typical example is a so-called transverse complex point (i.e., the point at which the tangent plane is a complex line and a certain mild transversality condition holds for the germ at this point) comprehensively studied by E. Bishop in [3]. The simplest model of such a germ is defined by the parametrization:

$$
(u, v) \mapsto\left(u, v,(1+2 \gamma) u^{2}+(1-2 \gamma) v^{2}+P_{3}(u, v), Q_{3}(u, v)\right),
$$

where $\gamma \neq \frac{1}{2}$ is a positive real number and $P_{3}, Q_{3}$ are some convergent series containing only terms of order not less than three. Such a point is called elliptic (resp. hyperbolic) if $\gamma<\frac{1}{2}$ (resp. $\gamma>\frac{1}{2}$ ).

Notice that the surface germ at a transverse complex point can be considered as the graph of a polynomial endomorphism of the plane $\mathbf{R}^{2}$ defined by the last two of the parametrizing polynomials above.

It can be shown that for a generic polynomial endomorphism of the plane, its graph defines a ROTORG as above. In such a situation we speak of a graph 
ROTORG (GROTORG). Such "palindromic" objects are especially convenient to work with and we will benefit from this circumstance in the sequel.

A rich source of ROTORGs is also given by the so-called totally real models of isolated singularities (TORMIS) of (pseudo-)holomorphic curves [11], in particular, one can construct such models for isolated singularities of plane algebraic curves [17], [11]. In [11] the existence of such models is derived from the transversality theory but in concrete cases one can effectively construct them as suitable small perturbations of a given singular germ. Notice that, unlike stable small deformations (e.g., morsifications) used in the singularity theory, these perturbations do not change the topological type of the singular point, they rather change the way it lies in $\mathbf{C}^{2}$. For such TORMISes, there apparently should exist connection between their topological invariants and algebraic invariants of the unperturbed ("generating") singularity. One application of this connection will be mentioned at the end of the section.

Remark 6. During his stay in Uppsala the author had an opportunity to discuss this issue with Norbert A'Campo who expressed an opinion that such totally real deformations can be useful for the study of monodromy of plane curve singularities [2]. Also, N. A'Campo showed how to construct such deformations in concrete examples in terms of Newton diagrams and then to investigate their geometric properties using the computer programs SINGULAR and SURF. His methods seem to admit formalization and there is good evidence that one can work out an algorithm applicable for any isolated singularity. In this way, totally real deformations of isolated singularities become related to some natural problems of the singularity theory.

We proceed by introducing basic topological invariants of totally real germs. We follow [15], [9] and perform some auxiliary geometric constructions. From the transversality condition in Definition 1, it follows that intersections $K_{t}=$ $X \cap S_{t}^{3}$ of $X$ with sufficiently small three-spheres around the basepoint define isotopic smooth links [2] which we will call links of $X$. We fix such a (small) link and denote it by $K$. It is clear that $K$ comes with the natural orientation induced from $S^{3}$ and $X$.

Take now any tangent vector field $V$ on $X$ which is directed outwards (with respect to $X$ ) on $K$ (such vector fields are easily seen to exist and below we will explain how one can effectively construct them in concrete examples). Since for any $p \in K$ the plane $T_{p} X$ is totally real, the field $\mathbf{i} V$ is transversal to $X$ along $K$. So a small perturbation $X+s \mathbf{i} V$ of $X$ is again transversal to $S=S_{t}^{3}$ and $K_{s}=\{X+s \mathbf{i} V\} \cap S$ is a smooth link in $S$ which does not intersect $K$.

Thus we obtain two oriented submanifolds $K$ and $K_{s}$ in $S^{3}$ and the sum of their dimensions is less by one than the dimension of the ambient manifold $S^{3}$. As is well known, in such a situation one can define the linking number of these two submanifolds which is homotopy invariant and possesses other nice properties [16]. For this reason, it does not depend on our (sufficiently small) $t, s$ and provides a numerical invariant of the germ $X$. 
Definition 2. With the same notation as above, the self-intersection index $i(X)$ of ROTORG $X$ is defined as the linking number $l\left(K, K_{s}\right)$ taken in $S_{t}^{3}$ for sufficiently small $t, s$.

The basic properties of the self-intersection index were established in [15] and [9]. This index describes some properties of smooth (small) perturbations of $X$ and is closely related to another numerical invariant of a typical TORG $X$ called Maslov index $m(X)$ [6], [9]. A pair of these invariants reflects some important geometric properties of the embedding of $X$ in $\mathbf{C}^{2}$. In particular, these invariants enable one to count different types of complex tangents in smooth perturbations of $X$.

In this paper we do not deal much with the Maslov index but, in virtue of the relations between $i(X)$ and $m(X)$ established in [9], our results actually imply some estimates for $m(X)$. So for the sake of completeness we present the definition of the Maslov index.

Consider the Gauss mapping of $X$ restricted to a (small) link $K$ as above:

$$
\Gamma: K \rightarrow G, \quad p \mapsto T_{p} X
$$

By the definition of a ROTORG, the image $\Gamma(K)$ lies in $G_{R}$ so it does not intersect $G_{C}$. Thus we obtain two oriented submanifolds of $G$ and the sum of their dimensions is again less by one than the dimension of the ambient manifold $G$. Thus one can define their linking number and conclude that it does not depend on the choice of a (sufficiently small) link $K$. In order that complex tangents could be counted properly, it is necessary to take the linking number after reversing the orientation of the second component $P_{-}$of $G_{C}$.

Definition 3. The Maslov index $m(X)$ of a ROTORG $X$ is defined as the linking number $l\left(\Gamma(K), P_{+} \cup\left(-P_{-}\right)\right)$taken in $G$ for a sufficiently small link $K$.

The most general way of computing both these invariants is to construct a proper (generic) deformation of $X$, count its complex tangents and selfintersections, and then apply the index formulas from [5] or [9].

For example, in the case of the unfolded Whitney umbrella $W$ it is sufficient to consider a simple deformation

$$
W_{t}(u, v)=\left(x^{2}+t x, y, x y, \frac{2 x^{3}}{3}\right), \quad 0<t \ll 1,
$$

which has no self-intersections and has only one complex point, i.e., of the hyperbolic type. Hence the formulas from Theorem 2 of [9] yield

$$
i(W)=-2, \quad m(W)=-1 .
$$

In [9] one can find some other examples of such computations.

Universal as it is, this method is by no means algorithmic and not always easy to implement in concrete examples. So it is important to develop more effective methods and it turns out that this can be done using the signature technique described in the previous section. Moreover, along the same lines it is possible to obtain those algebraic estimates which are our main concern in 
this paper. In the next section we explain how all this works in the case of a self-intersection index.

Concluding this section, we would like to add that these two invariants are not independent. For example, their parities (classes mod2) always differ by one. The formulas expressing relation between $i(X)$ and $m(X)$ are presented in [9]. So our estimates for $i(X)$ in certain situations can provide estimates for $m(X)$ as well.

For example, for TORMISes arising as totally real perturbations of a plane curve singularity of degree $d$, it is possible to show that they always have generic deformations without self-intersections, while the numbers of nearby complex tangents of arbitrary type are bounded by some explicit function of $d$ (because complex points emerge as solutions of a system of polynomial equations with degrees derivable from $d[11])$. So our bounds for $i(X)$ also imply certain bounds for $m(X)$. Granted that, a natural problem becomes to establish what pairs of values within these bounds can be actually attained by invariants of curves of fixed degree $d$. We hope that some light on this question may be shed by using the signature technique.

\section{Estimating Self-Intersection Index}

We are now in a position to obtain the desired estimate. For simplicity, we present the result in the case of a GROTORG $X$ and then briefly explain how one can extend it to more general situations.

Theorem 2. Let $X$ be a GROTORG in $\mathbf{C}^{2}$ defined as the graph of a generic $\left(w_{1}, w_{2} ; d_{1}, d_{2}\right)$-quasi-homogeneous endomorphism of $\mathbf{R}^{2}$. Then one has the estimate

$$
|i(X)| \leq P\left(4 ;\left(w_{1}, w_{2}, w_{1}, w_{2}\right),(W, W, D, D)\right),
$$

where $W=\min \left\{w_{1}, w_{2}\right\}, D=\min \left\{d_{1}, d_{2}\right\}$.

Proof. We will reduce the problem to estimating of the index of an auxiliary vector field. This becomes possible due to the following construction going back to $[9]$.

Denote by $f$ the defining endomorphism of the plane. Then it is easy to see that in our case the vector field $V$ involved in the definition of $i(X)$ can be obtained by setting $V=f_{*}(E)$, where $E$ is the Euler vector field $E=u \partial_{u}+v \partial_{v}$. So it becomes clear that in our case an appropriate deformation $K_{s}$ of $K$ can be constructed as the image of a mapping $f_{s}$ obtained from $f$ by adding a small multiple $s \mathbf{i} V$ of the rotated vector field $\mathbf{i} V$. It is then easy to check that $f_{s}$ is semi-quasi-homogeneous mapping of $\left(w_{1}, w_{2}\right)$-multi-degree $(W, W, D, D)$, with $W$ and $D$ such as indicated in the formulation of theorem.

Now, it is a well known topological fact that linking numbers can be interpreted in terms of the mapping degree or indices of vector fields [16]. In our 
case this general circumstance implies that the linking number $l\left(K, K_{s}\right)$ can be interpreted as the local index of a polynomial vector field

$$
F: \mathbf{R}^{4} \rightarrow \mathbf{R}^{4}, F(x, y, u, v)=f_{s}(u, v)-f(x, y) .
$$

Since the components of such $F$ are just differences of the corresponding components of $f$ and $f_{s}$, it is easy to estimate $\left(w_{1}, w_{2}\right)$-degrees of their summands and see that $F$ can be considered as a semi-quasi-homogeneous vector field of multi-degree $(W, W, D, D)$ with respect to the weights $\left(w_{1}, w_{2}, w_{1}, w_{2}\right)$. So the desired estimate follows directly from Theorem 1.

In the same way one can get similar estimates for an arbitrary parametrized ROTORG if the qh-type of its parametrization is known, for example if it is given by an explicit parametrization. As is well known, one can also obtain information about the qh-type of leading terms of parametrizations of ROTORGs defined as zeroes of polynomials systems of the known qh-type, by using the technique of Newton diagrams and Puiseaux expansions [2]. So similar estimates are available for ROTORGs defined as zeroes of qh-polynomials.

At the moment we cannot make any comments about the exactness of such an estimate, but we believe that this issue can be successfully investigated using the same approach. As has already been mentioned, it is also interesting to investigate what values can be taken by $i(X)$ and $m(X)$ for ROTORGs with the fixed qh-type.

Another interesting perspective is related to numerical invariants of totally real germs in higher dimensions. Apparently the self-intersection index can be defined for a totally real manifold of arbitrary dimension in the same way as above. Other local numerical invariants available in this case can be constructed using the results of [13]. Apparently, using our approach one can obtain similar estimates for the self-intersection index for arbitraty dimensions. One can also try to generalize our estimates to the case of totally real surfaces with robust singularities lying in an (almost) complex four-dimensional manifold, in the spirit of [15] and [9].

Tempting as they are, we delay these and other possible generalizations till forthcoming publications.

\section{ACKNOWLEDGEMENTS}

These results were obtained during a long-term visit of the author to the Department of Mathematics of Uppsala University in the framework of a scholarship provided by the Swedish Institute. The author is grateful to Prof. C. Kiselman for warm hospitality and permanent support. Useful discussions with Prof. O. Viro and Prof. N. A'Campo are also acknowledged.

\section{REFERENCES}

1. V. I. Arnol'D, Index of a singular point of a vector field, Petrovsky-Oleynik inequalities, and mixed Hodge structures. (Russian) Funktsional. Anal. i Prilozhen. 12(1978), 1-14. 
2. V. I. Arnol'd, A. N. Varchenko, and S. M. Gusein-Zade, Singularities of differentiable mappings. I. (Russian) Nauka, Moscow, 1982.

3. E. BIshop, Differentiable manifolds in complex euclidean space. Duke Math. J. 32(1965), $1-21$.

4. D. Eisenbud and H. Levine, An algebraic formula for the degree of a $C^{\infty}$ map-germ. Ann. of Math. (2) 106(1977), 19-38.

5. F. Forstneric, Complex tangents of real surfaces in complex surfaces. Duke Math. J. 67(1992), 353-376.

6. A. B. Givental, Lagrangian embeddings of surfaces and unfolded Whitney umbrella. (Russian) Funktsional. Anal. i Prilozhen. 20(1986), 35-41.

7. G.-M.Greuel and H. Hamm, Invarianten quasihomogener Durchschnitte. Invent. Math. 49(1978), 67-86.

8. P. Griffiths and J. Harris, Principles of algebraic geometry. J. Wiley, New York, 1978.

9. G. Ishikawa and T. Онмото, Local invariants of singular syrfaces in an almost complex four-manifold. Ann. Global Anal. Geom. 11(1993), 125-133.

10. G. N. Khimshiashvili, On the local degree of a smooth mapping. (Russian) Bull. Acad. Sci. Georgian SSR 85(1977), 309-312.

11. G. N. Khimshiashvili and E. Wegert, Analytic discs and totally real surfaces. Bull. Georgian Acad. Sci. 162(2000), 41-44.

12. A. G. Khovanskit, Index of a polynomial vector field. (Russian) Funktsional. Anal. $i$ Prilozhen. 13(1979), 49-58.

13. H.-F. LAI, Characteristic classes of real manifolds immersed in complex manifolds. Trans. Amer. Math. Soc. 172(1972), 1-33.

14. A. Lecki and Z. Szafraniec, An algebraic method of computing topological degree. Gdansk Univ. Preprint, Gdansk, 1993.

15. D. McDuff, The local behaviour of holomorphic curves in almost complex fourmanifolds. J. Differential Geom. 34(1991), 143-164.

16. E. Spanier, Algebraic topology. McGraw-Hill, New York, 1965.

17. E. Wegert, G. Khimshiashvili, and I. Spitkovsky, Nonlinear transmission problems. Mem. Differential Equations Math. Phys. 12(1997), 223-230.

(Received 22.09.2000)

Author's address:

A. Razmadze Mathematical Institute

Georgian Academy of Sciences

1, M. Aleksidze St., Tbilisi 380093

Georgia

E-mail: khimsh@rmi.acnet.ge 